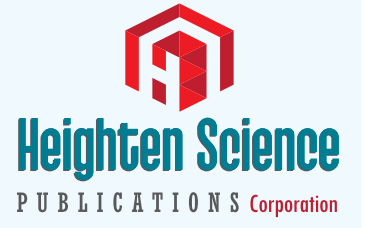

ISSN

2640-2890

\title{
Utilization of post abortal contraceptive use and associated factors among women who came for abortion service at Debre Berhan Hospital, Debre Berhan, Ethiopia March 2019: Institution based cross sectional
} study

\author{
Abebe Muche ${ }^{1 *}$, Bekalu Bewket ${ }^{2}$, Eferem Ayalew ${ }^{3}$ and Endale \\ Demeke $^{3}$ \\ 'Lecturer of Biochemistry, Department of Medicine, College of Medicine, Debere Berhan \\ University, Debere Berhan Ethiopia \\ 2Lecturer of Adult Health Nursing, Department of Nursing, College of Health Science, Debre \\ Markos University, Debre Markos, Ethiopia \\ ${ }^{3}$ General Practitioner, Deber Berhan Hospital, Debre Berhan, Ethiopia
}

*Address for Correspondence: Abebe Muche, Lecturer of Biochemistry, Department of Medicine, College of Medicine, Debere Derhan University, Debere Berhan, Ethiopia, Tel: +25143446400; Email: abebemuche3@gmail.com; bewket.bekalu2014@gmail.com

Submitted: 13 June 2019

Approved: 19 June 2019

Published: 20 June 2019

Copyright: @ 2019 Muche A, et al. This is an open access article distributed under the Creative Commons Attribution License, which permits unrestricted use, distribution, and reproduction in any medium, provided the original work is properly cited

Abbreviations: Cl: Confidence Interval; DBRH: Debre Birhan Referral Hospital; DBU: Debire Birhan University; FP: Family Planning; HCG: Human Chorionic Gonadotropic Hormone; HIP: High Impact Practice; IUCD: Intra Uterine Contraceptive Device; MMR: Maternal Mortality Rate; MOH: Ministry of Health; MSC: Marie Stop Clinic; NGO: Non-Governmental Organization; PAC: Post Abortion Care; PAFP: Post Abortion Family Planning; SAC: Safe Abortion Care; SPSS: Statistical Package for Social Science; SRS: Systematic Random Sampling; WHO: World Health Organization

Check for updates

\section{Abstract}

Background: Post abortion family planning (PAFP) is the initiation and use of family planning methods immediately after, and within 48 hours of an abortion, before fertility returns. In most women fertility returns on average about two weeks after an abortion; however, ovulation can occur as early as 11 days post-abortion.

Objective: To assess utilization of post abortal contraceptive use and associated factors among women who came for abortion service at Debre Berhan Referral Hospital, Debre Berhan, Ethiopia March 2019.

Methodology: Institutional based cross sectional study design was conducted using hospital data obtained from Gynecology ward in Debre Berhan Referral Hospital, Debre Berhan, Ethiopia, from March $1-10,2019$. A systematic random sampling technique was used from the abortion register log book. Data was cleaned manually, coded and entered into Epi-data version 3.1 then exported to and analyzed by SPSS version 21 software. Multivariate analysis with AOR, $95 \% \mathrm{Cl}$ and $p$-value $<0.05$ were used to identify variables which have significant association.

Result: The finding of the current study showed that among 371 study subjects $170(45.8 \%)$ utilized post abortal family planning. There was a significant association between utility of post abortal family planning and post abortion family planning counseling [AOR: $19.245,95 \% \mathrm{Cl}$ : $(10.199,36.313), p$-value $=0.001]$ and women who were primiparous had 5 times more likely to utilize post abortal family planning as compared to the women who were nullyparous [AOR: 5.314, $95 \% \mathrm{Cl}(1.089,24.210)$, $\mathrm{p}$ value $=0.001]$.

Conclusion and Recommendation: From a total of 371 study subjects $45.8 \%$ have utilize contraceptive after abortion service received. This study also showed that parity and counseling's of family planning were statistically significant associated with utilization of post abortal family planning. We recommend Debre Berhan hospital to scale up activities on post abortal care to increase the number of clients who post abortal family planning. 


\section{Introduction}

Post abortion family planning (PAFP) is the initiation and use of family planning methods immediately after, and within 48 hours of an abortion, before fertility returns. In most women fertility returns on average about two weeks after an abortion; however, ovulation can occur as early as 11 days post-abortion [1].

Abortion is defined as the spontaneous or induced termination of pregnancy before fetal viability. It thus is appropriate that miscarriage and abortion are terms used interchangeably in a medical context. But because popular use of abortion by laypersons implies a deliberate intact pregnancy termination, many prefer miscarriage for spontaneous fetal loss [2].

One can presume that abortion is most often chosen as a response to a crisis or unintended pregnancy. The high prevalence of a history of induced abortion means that even small positive or negative effects on long-term health could influence the lives of many women and their families [3].

PAFP has been implemented in countries using two main methodologies. In countries where abortion is legal, programs offer post abortion family planning only. When women make an appointment for their abortion, they also receive family planning counseling. After their abortion has been completed, women are provided with family planning services before discharge from the facility [4].

Women who receive post abortal care (PAC) without the necessary tools or information needed to prevent subsequent unwanted pregnancies and abortions may find themselves returning to health centers for similar services in the future. Lack of family planning information and tools leave women trapped in what has been called a harmful cycle of unwanted pregnancy and unsafe abortion. Research shows that reaching women at this critical stage helps to increase contraceptive use significantly, leading to fewer repeat and possibly unsafe abortions [5].

Women who receive PAC without the necessary tools or information needed to prevent subsequent unwanted pregnancies and abortions may find themselves returning to health centers for similar services in the future. Lack of family planning information and tools leave women trapped in what has been called a harmful cycle of unwanted pregnancy and unsafe abortion. Research shows that reaching women at this critical stage helps to increase contraceptive use significantly, leading to fewer repeat and possibly unsafe abortions [6].

Unsafe abortion is a major public health problem in many countries. It is one of the most easily preventable causes of maternal death and ill-health which causes about $13 \%$ of global maternal mortality [7].

\section{Methods}

\section{Study area}

Debire Birhan city is the capital city of North showa zone which is located $130 \mathrm{Km}$ away from Addis Ababa, the capital city of Ethiopia. According to Wikipedia itlies in at an altitude of 2840 meters. The city has surface area of about $14.71 \mathrm{~km}^{2}(5.68 \mathrm{sq}$. mi). It has a total population of 65,231 , of whom 31,668 are men and 33,563 women. The majority of the inhabitants practiced Ethiopian Orthodox Christianity, with $94.12 \%$ reporting that as their religion, while $3.32 \%$ of the population said they were Muslim and $2.15 \%$ were Protestants. Debre Birhan hospital is the only referral hospital in the city and was found in 1929 E.C during Italian colonization. Now it is believed that it serves around 2.8 million people and organized with around 516 workers and 150 inpatient beds. 


\section{Study design}

Facility based descriptive cross sectional study design from a secondary data was used.

\section{Population}

Source population: all women who came for abortion service at Debire Birhan referral hospital.

Study population: Those who got abortion service in the time period and fulfill the inclusion criteria.

\section{Inclusion criteria}

Those who were documented in the abortion register during the time period were included in the study period.

\section{Exclusion criteria}

Those women who were registered in the abortion register with incomplete data were excluded from the study.

\section{Sample size calculation}

The required sample size was determined by using single population proportion formula i.e

$$
\mathrm{N}=\frac{\mathrm{z}_{*}^{2} \mathrm{p}(1-\mathrm{p})}{\mathrm{d}^{2}}
$$

Where: $\mathrm{N}=$ desired sample size

$\mathrm{Z} / 2$ = the value of $\mathrm{Z}$ in SND that corresponds to level of 0.05

$\mathrm{P}=$ Family Planning of PAFP study done in Debire Markos Ethiopia $=59.2 \%$ [8]

$q=1-p=40.8 \% d=$ degree of precision $=5 \%$

$\mathrm{N}=(1.96)^{2 *} 0.59 * 0.41$

$$
(0.05)^{2}
$$

$\mathrm{N}=371$ Hence, the calculated sample size is 371

\section{Sampling technique and procedure}

Simple random sampling was employed to select the required number of subjects using medical registered number as a sampling unit.

\section{Study variables}

The dependent variable of the study was Post abortal family planning utilization and independent variables were Socio-demographic characteristics (age, parity, gravidity); previous history variables (number of previous abortion history); Present history variables (gestational age, post abortion counseling, reason for current abortion, type of procedure done) and Complication after procedure.

\section{Data collection procedure and tool}

The data was collected from the hospital within 2 months by 2 clinical nurses. The study was controlled by 2 BSC nurses.

A structured check list was used as a data collection tool. The data collection was mainly focused on the objective of the study. All necessary information was collected from the register which consists of socio-demographic, parity, gravidity and previous 
history of abortion, questions related to acceptance of post abortion family planning and post procedure complications.

\section{Data processing and analysis}

Data was checked, cleaned and entered into Epi -data version 3.1 software. Then it was exported to SPSS version 21 for analysis. Descriptive statistics was used to describe the sample. The results of the descriptive statistics were expressed as percentage and frequency. Associations between independent variables and dependent variables were analyzed first by using bivariate analysis to identify factors which are significantly associated with the outcome variable. Those variables with p-value less than or equal to 0.05 became candidate for multivariate analysis. The magnitude of the association between the different independent variables in relation to dependent variables were measured using odds ratios and 95\% CI and P-values below 0.05 will be considered statistically significant.

\section{Result}

\section{Socio demographic characteristics}

A total of three hundred seventy-one (371) women who come for abortion service data were included I the study collected from Debre Berhan referral hospital. The majority of women's are between the ranges of 20-29 years old with mean age of 27.99 years.

According to gestational age 126/370(34.0\%) were less than nine week, $132 / 371(35.6 \%)$ were between $9-12$ weeks and 113/371(30.5\%) were greater than 12 weeks. From the total of 371 women's come for abortion service 256/371(69.0\%) were between gravida 2- 4. Regarding the parity from the total 371 women $88 / 371(23.7 \%)$ were para zero, 99/371(26.7\%) were para one and $109 / 371(29.4 \%)$ were para two (Table 1).

\section{Abortion and related factors}

In the present study, 57/371(15.4\%) were having history of previous abortion. Of those 45/57(78.9\%) were having one previous history of abortion. Considering types of abortion performed353/371(95.1\%) was complicated abortion. Among women's who had safe abortion 3/18(16.7\%) were for rape and 15/18(83.3\%) were for abnormal fetal condition, as an indication for abortion. From 353 women's who come for complicated abortion 218/353(61.8\%) were for incomplete abortion, $80 / 353(22.7 \%)$ and were for missed abortion (Table 2). For 338/371(91.1\%) were undergo manual vacuum aspiration as means of uterine evacuation (Table 2).

\section{Post abortal FP counseling and utilization}

In the current study, 203/371(54.3\%) study subjects were counseled for PAFP. Among these, 170/371(45.8\%) of them were received the contraceptive after abortion service. Regarding to the type of contraceptive used, 82/170(48.2\%) were given implants and 43/170(25.3\%) were given DEPO (Table 3).

\section{Factors associated post abortive FP utilization}

Bivariable logistic regression test was carried out to determine any association between utilization of PAFP contraceptive and the independent variables. Bivariate analysis showed that there were significant association between utilization of PAFP contraceptive and age, gravidity, PAFP counseling and parity of study subjects.

Multivariate analysis revealed that there was statistical significant association between utilization of PAFP with counseling and increment in Parity. Women who were primiparous had 5 times more likely to utilize PAFP as compared to the women who were nullyparous [AOR: 5.314, 95\%CI (1.089, 24.210)]. In addition, those para 
two and above were 32 times more likely to utilize PAFP contraceptive than women who were nullyparous [AOR: $31.74295 \% \mathrm{CI}(6.470,155.731)]$. Study subjects received post abortion contraceptive counseling were 19 times more likely to utilize PAFP as compared to women who did not get the post abortion contraceptive counseling [AOR: 19.245, 95\% CI: (10.199, 36.313)] (Table 4).

\section{Discussion}

Worldwide, 210 million women become pregnant each year. Of these, 80 million pregnancies are unplanned. Out of these, 46 million pregnancies terminated each year, and 19 million ends with unsafe abortion. More than $97 \%$ of unsafe abortions take

Table 1: Reproductive characteristics among women who came for abortion service at Debre Berhan Referral Hospital Debre Berhan, Ethiopia March 2019.

\begin{tabular}{|c|c|c|c|}
\hline Variable & Category & Frequency & Percent \\
\hline \multirow{4}{*}{ Gestational age } & $<9$ WKS & 126 & 34.0 \\
\cline { 2 - 4 } & $9-12$ WKS & 132 & 35.6 \\
\cline { 2 - 4 } & $>12$ WKS & 113 & 30.5 \\
\hline \multirow{4}{*}{ Gravidity } & Total & $\mathbf{3 7 1}$ & $\mathbf{1 0 0 . 0}$ \\
\hline \multirow{5}{*}{} & 1 & 79 & 21.3 \\
\hline \multirow{4}{*}{ Parity } & $2-4$ & 256 & 69.0 \\
\hline & $>=5$ & 36 & 9.7 \\
\hline & Total & $\mathbf{3 7 1}$ & $\mathbf{1 0 0 . 0}$ \\
\hline & Nulliparous & 88 & 23.7 \\
\hline & Primiparous & 99 & 26.7 \\
\hline
\end{tabular}

Table 2: Abortion and related issues among women who came for abortion service at Debre Berhan Referral Hospital Debre Berhan, Ethiopia March 2019.

\begin{tabular}{|c|c|c|c|}
\hline Variable & Category & Frequency & Percentage \\
\hline \multirow{3}{*}{ History of abortion } & Yes & 57 & 15.4 \\
\hline & No & 314 & 84.6 \\
\hline & Total & 371 & 100.0 \\
\hline \multirow{4}{*}{ Number of previous abortion } & 1 & 45 & 78.9 \\
\hline & 2 & 9 & 15.8 \\
\hline & $\geq 3$ & 3 & 5.3 \\
\hline & Total & 57 & 100.0 \\
\hline \multirow{3}{*}{ Types of abortion offered } & Safe & 18 & 4.9 \\
\hline & For complicated & 353 & 95.1 \\
\hline & Total & 371 & 100.0 \\
\hline \multirow{3}{*}{ Reason for safe abortion } & Rape & 3 & 16.7 \\
\hline & Fetal condition & 15 & 83.3 \\
\hline & Total & 18 & 100.0 \\
\hline \multirow{5}{*}{ Type of complicated abortion } & Incomplete & 218 & 61.8 \\
\hline & Missed & 80 & 22.7 \\
\hline & Inevitable & 21 & 5.9 \\
\hline & Others & 34 & 9.6 \\
\hline & Total & 353 & 100.0 \\
\hline \multirow{3}{*}{ Method of uterine evacuation } & MVA & 338 & 91.1 \\
\hline & MA & 33 & 8.9 \\
\hline & Total & 371 & 100.0 \\
\hline
\end{tabular}

Table 3: Post abortal FP counseling and utilization among women who came for abortion service at Debre Berhan Referral Hospital Debre Berhan, Debre Berhan, Ethiopia, March 2019.

\begin{tabular}{|c|c|c|c|}
\hline Variable & Category & Frequency & Percent \\
\hline \multirow{3}{*}{$\begin{array}{c}\text { Counseled for post abortal } \\
\text { contraceptive }\end{array}$} & Yes & 203 & 54.7 \\
\cline { 2 - 4 } & No & 168 & 45.3 \\
\cline { 2 - 4 } & Total & $\mathbf{3 7 1}$ & $\mathbf{1 0 0 . 0}$ \\
\hline \multirow{3}{*}{ Type of contraceptive given } & Implant & 82 & 48.2 \\
\cline { 2 - 4 } & DEPO & 43 & 25.3 \\
\cline { 2 - 4 } & COCs & 32 & 18.8 \\
\hline & IUCD & 13 & 7.6 \\
\hline
\end{tabular}


Table 4: Bivariate and multivariate analysis between utilization of PAFP contraceptive and the independent variables of women who receive abortion service at Debre Berhan Referral Hospital, Debre Berhan, Ethiopia March 2019.

\begin{tabular}{|c|c|c|c|c|c|c|c|}
\hline \multirow{2}{*}{ Variable } & \multirow{2}{*}{ Category } & \multicolumn{6}{|c|}{ Utilization of PAFP contraceptive } \\
\hline & & Yes & No & COR(95\%Cl) & $P$-value & AOR(95\%Cl) & $P$-value \\
\hline \multirow{5}{*}{ Age } & $15-19$ & 3 & 11 & 1 & & & \\
\hline & $20-24$ & 28 & 59 & $1.740(0.450,6.736)$ & 0.4 & $0.424(0.091,1.979)$ & 0.275 \\
\hline & $25-29$ & 62 & 77 & $2.952(0.789,11.048)$ & 0.108 & $0.287(0.58,1.416)$ & 0.125 \\
\hline & $30-34$ & 45 & 28 & $5.893(1.511,22.982)$ & 0.011 & $0.335(0.060,1.881)$ & 0.214 \\
\hline & $\geq 35$ & 32 & 26 & $4.513(1.138,17.893)$ & 0.032 & $0.209(0.035,1.269)$ & 0.089 \\
\hline \multirow{3}{*}{$\begin{array}{l}\text { Gestational age } \\
\quad \text { (in weeks) }\end{array}$} & $<9$ wks. & 69 & 57 & 1 & & & \\
\hline & $9-12$ wks. & 55 & 77 & $0.590(0.360,0.966)$ & 0.036 & & \\
\hline & $>12$ wks. & 46 & 67 & $0.567(0.339,0.948)$ & 0.030 & & \\
\hline \multirow{3}{*}{ Gravidity } & 1 & 10 & 69 & 1 & & & \\
\hline & $2-4$ & 143 & 113 & $8.732(4.303,17.718)$ & 0.001 & $1.649(0.341,7.981)$ & 0.534 \\
\hline & $\geq 5$ & 17 & 19 & $6.174(2.432,15.673)$ & 0.001 & $0.669(0.104,4.307)$ & 0.672 \\
\hline \multirow{3}{*}{ Parity } & 0 & 11 & 77 & 1 & & & \\
\hline & 1 & 34 & 65 & $3.662(1.720,7.796)$ & 0.001 & $5.134(1.089,24.210)$ & $0.04^{*}$ \\
\hline & $\geq 2$ & 125 & 59 & $14.831(7.339,29.971)$ & 0.001 & $31.742(6.470,155.731)$ & $0.001 *$ \\
\hline \multirow{2}{*}{ History of abortion } & No & 29 & 172 & 1 & & & \\
\hline & Yes & 28 & 142 & $1.169(0.665,2.057)$ & 0.6 & & \\
\hline \multirow{2}{*}{ Post abortion counseling } & No & 60 & 141 & 1 & & & \\
\hline & Yes & 143 & 27 & $12.446(7.472,20.733)$ & 0.001 & $19.245(10.199,36.313)$ & $0.001 *$ \\
\hline
\end{tabular}

place in developing countries. WHO estimates that one in ten pregnancies end up with unsafe abortion, giving one unsafe abortion to seven live births ratio. Likewise, 68,000 women die due to unsafe abortion each year, and the risk of maternal death is high in developing countries (1 in 270 unsafe abortions) [6]. In Ethiopia Between 2008 and 2014 , the use of appropriate technology for conducting first and second trimester abortion and the provision of post abortion family planning has increased at the same time that abortion-related obstetric complications have decreased [9].

The present study assessed utilization of PAFP contraceptive among 371 women who receive abortion service and associated factors of utilization of PAFP at Debre Berhan Referral Hospital. Data coming from this study ascertained that $37.5 \%$ of the study subjects were with in age of $25-29$ years, $35.6 \%$ were between $9-12$ weeks of gestational age, $69.0 \%$ were between gravida $2-4$, and $29.4 \%$ of them were para two. $15.4 \%$ of the clients were having history of previous abortion. Currently only $4.9 \%$ of them undergo safe abortion, with the most common indication being abnormal fetal condition which accounts $83.3 \%$. $45.8 \%$ of study subjects got some form of family planning method of which $48.2 \%$ took implants.

The results of current study revealed that $61 \%$ were in the age of $20-29$. This result is similar with a study done in Debre Markos Ethiopia (67.9\% within the age of 20-29) [10], and Gondar Ethiopia (67.2) [11]. 26.7\% of the study subjects were primiparous (Table 1) but a study done in Nepal shows $47.4 \%$ and Debre Markos shows $72 \%$ $[10,12]$. The difference might be accounted for sample size variation (3190 and 414 respectively) and study design 00000000000000000000000 (population based in the case of Nepal).

Considering history of previous abortion $15.4 \%$ of the study subjects were having history of previous abortion. This result was similar with the study conducted in Gondar-Ethiopia (14.4\%), Debre Markos-Ethiopia(12.3\%) but it was lower than the prevalence reported from the study conducted in Jimma-Ethiopia which was 30.7\% $[8,10,11]$. This could be due to sample size variation (204) and study period (2015).

Out of 371 client $54.7 \%$ were counseled for utilization of post abortal contraceptive. This figure is also comparable with a study done at Debire Markos-Ethiopia (52.9\%) but lower than a study done at Tigray region -Ethiopia $(97.8 \%)[10,13]$. This might be due to larger sample size (416) and study involved different health institutions. 
In the current study PAFP was given for $45.8 \%$ clients. This figure is comparable with studies done at Gondar- Ethiopia (55.4\%) and Debre Markos-Ethiopia (59.2\%) $[10,11]$. But it was lower than the prevalence reported from the study conducted in Pakistan (73\%), Kenya (75\%) and Tigray region $(70.9 \%)$. This might be due to difference in sample size variation (17267, 859 and 416 respectively) [13-15]. Out of 170 PAFP users $48 \%$ were using implants. This is similar with studies done in JimmaEthiopia (54\%) and Gondar Ethiopia (44\%) [8,11].

The current study had investigated the associations of some demographic, reproductive, abortion history, parity, counseling of PAFP and utilization of PAFP. PAFP counseling of the client has association with utilization of PAFP [(AOR=19.245), 95\% CI (10.199- 36.313), p-value=0.001] in comparison with those not counseled. Regarding to PAFP counseling the result was found to be similar with studies conducted at Gambella-Ethiopia and Debre Markos $[10,16]$. This might be due to similarity in study site (institution) and study design.

The study shows a statistically significant association between being primiparous [(AOR=5.134), 95\%CI $(1.089,24.210), \mathrm{p}$-value=0.04] and PAFP when compared with that of nulliparous. A study done in Angola showed that no association between primparity and PAFP utilization [AOR=3.52, 95\%CI $(0.59,20.92)$, p-value=0.167] which contradicts with possible reason of difference in study period (January 2012), sample size (1176) and sampling technique (multistage) [12].

Other variables fail to show any significant association with utilization of PAFP, for instance gravidity greater or equal to 2 [AOR=0.669, 95\%CI (0.014-4.307), $\mathrm{p}$-value $=0.587]$. This result is differ from the study done at Gondar-Ethiopia which showed that there is a significant association between them. The reason might be due to larger sample size in the study done in Gondar which is 662 and study period (2013) [11].

- Strength of the studyThe study can be an entry point for other studies in the vicinity.

- The study can be used as a planning tool so as to improve activities in the hospital related and regarding to PAFP counseling and use.

- Parity and gestational age was assessed for its association to PAFP utilization.

\section{Limitation of the study}

- As being a secondary data variables were limited

- It may fail to show cause and effect

- The way things measured may change over time making historical comparisons difficult.

- Documents may not be representative of the wider population since the study involves only one hospital.

\section{Conclusion}

The result of the present study evaluated utilization of PAFP and its associated factors. Our research found that $45.8 \%$ of the study subjects utilized PAFP after abortion service received. This study also showed that increment in parity and counseling for PAFP were found statistically significant association with utilization of PAFP. Other variables were not found statistically significantly.

\section{Recommendation}

Debre Berhan Referral Hospital might scale up activities on PAC to increase the number of clients who utilize post abortal contraceptives. 
Service providers should provide brief information about fertility return following abortion and detail counseling on PAFP.

Service providers have to get training on comprehensive post abortion care and on integration of family planning services.

\section{Declaration}

\section{Ethical approval and consent to participate}

Ethical clearance was obtained from Ethical Review Committee of the School of Public Health of Debire Birhan University and permission was taken from Debire Birhan Hospital. Names or any identification never been used. Concerning supplies and equipment assessment, official letter from North showa health bureau permission was requested from service provider institutions.

\section{Availability of data and materials}

"The dataset will not be shared in order to protect the participants' identities"

\section{Author's contribution}

AA conceived and designed the study, performed analysis and interpretation of data. AS and BB advised and supervised the design conception, analysis, interpretation of data and made critical comments at each step of research. EA and ED drafted the manuscript. All authors read and approved the final Manuscript.

\section{Acknowledgment}

We would like to thank data collectors and supervisors for their great contribution and timely submission of filled questioners. Our sincere thanks also goes to Debre Berhan Referral hospital administrative staffs for their permission to participate in this thesis work. We want to extend our heartfelt gratitude for Debre Berhan University for giving us all the necessary and available services to conduct this study.

\section{References}

1. World Health Organization (WHO). Post-abortion family planning: a practical guide for program managers. Geneva: WHO; 1997; Ref.: http://bit.ly/2RpdbuF

2. Cunningham G, Leveno KJ, Bloom SL, Spong CY, Dashe JS, et al. Williams Obstetrics. $24^{\text {th }}$ edition. New York. McGraw-Hill. 2014; 350-370. Ref.: http://bit.ly/2KW2IFY

3. Thorp JM Jr, Hartmann KE, Shadigian E. Long-Term Physical and Psychological Health Consequences of Induced Abortion. A Review of the Evidence the Linacre Quarterly. 2005; 72: 9: 1-27. Ref.: http://bit.ly/2lsEe5F

4. Curtis C, Huber D, Moss-Knight T. Postabortion Family Planning: Addressing the Cycle of Repeat Unintended Pregnancy and Abortion. IpS Reproductive Health. 2010; 36: 44-48. Ref.: http://bit.ly/2x6VgzM

5. Thapa S, Rani A, Mishra C. Knowledge, attitude and belief about contraception in post-partum and post abortal women in a tertiary care center. IJRCOG. 2014; 3: 533-539. Ref.: http://bit.ly/2lqR6sW

6. Merhawi G, Agumasie S, Tofik U, Gezahegn T. Unsafe abortion and associated factors among reproductive aged women in Sub-Saharan Africa: a protocol for a systematic review and metaanalysis. Open access. 2018; 1-5. Ref.: http://bit.ly/2RprfEk

7. Fact sheet, abortion in Africa, Guttmacher Institute. 2018; 1-4.

8. Erko EK, Abera M, Admassu B. Safe Abortion Care, Utilization of Post Abortion Contraception and associated Factors, Jimma Ethiopia. J Women's Health Care. 2016; 5: 1-12. Ref.: http://bit.ly/2IQBwpq

9. Dibaba Y, Dijkerman S, Fetters T, Moore A, Gebreselassie H, et al. A decade of progress providing safe abortion services in Ethiopia: results of national assessments in 2008 and 2014. BMC Pregnancy and Childbirth. 2017; 17: 1-12. Ref.: http://bit.ly/2FmNS7G

10. Kokeb L, Admassu E, Kassa H, Seyoum T. Utilization of Post Abortion Contraceptive and Associated Factors among Women who came for Abortion Service: a Hospital Based Cross Sectional Study. J Fam Med Dis Prev. 2015; 1: 1-4. Ref.: http://bit.ly/2Y01Un3 
11. Seyoum D, Gebeyehu A, Gizaw Z. Assessment of Post Abortion Contraceptive Intention and Associated Factors among Abortion Clients in Gondar Town, North West Ethiopia. Uni J Public Health. 2014; 2: 215-225. Ref.: http://bit.ly/31E8JNv

12. Shrestha A, Sharma P. Post Abortion Choice and Acceptance of Contraception Patan, Nepal. NJOG. 2013; 8: 1-4. Ref.: http://bit.ly/2ZBIQNE

13. Hagos G, Tura G, Kahsay G, Haile K, Grum T, et al. Family planning utilization and factors associated among women receiving abortion services in health facilities of central zone towns of Tigray, Northern Ethiopia: a cross sectional Study. BMC women Health. 2018; 18: 83. Ref.: http://bit.ly/2IU33Gy

14. Azmat $S$, Hameed W, Ishaque M, Mustafa G, Ahmed A. Post-abortion care family planning use in Pakistan. Pak J Public Health. 2012; 2: 4-9. Ref.: http://bit.ly/2IXkBBo

15. Makenzius M, Faxelid E, Gemzell-Danielsson K, Odero TMA, Klingberg-Allvin M, et al. Contraceptive uptake in post abortion care Secondary outcomes from a randomized controlled trial, Kisumu, Kenya. PLoS One. 2018; 13: e0201214. Ref.: http://bit.ly/2Y9MdcU

16. Abamecha A, Shiferaw A, Kassaye A. Assessment of Post Abortion Contraceptive Intention and Associated Factors among Abortion Clients In Gambella Health Facilities, Gambella Town, South West Ethiopia. Int J Med Sci Clini Inventions. 2016; 3: 2061-2070. Ref.: http://bit.ly/2WXmx28 